\title{
Interleukin-32 regulates downstream molecules and promotes the invasion of pancreatic cancer cells
}

\author{
KOHJI TAKAGI $^{1}$, AKIKO SHIMOMURA ${ }^{1}$, JOHJI IMURA ${ }^{1}$, HISASHI MORI ${ }^{2}$, AKIRA NOGUCHI ${ }^{1}$, SHINICHI TANAKA ${ }^{1}$, \\ TAKASHI MINAMISAKA ${ }^{1}$, TAKESHI NISHIDA ${ }^{1}$, HIDEKI HATTA $^{1}$ and TAKAHIKO NAKAJIMA ${ }^{1}$ \\ Departments of ${ }^{1}$ Diagnostic Pathology and ${ }^{2}$ Molecular Neuroscience, Faculty of Medicine, \\ Academic Assembly, University of Toyama, Toyama 930-0194, Japan
}

Received May 23, 2021; Accepted September 29, 2021

DOI: $10.3892 / \mathrm{ol} .2021 .13132$

\begin{abstract}
Pancreatic cancer is a malignant neoplasm with high invasiveness and poor prognosis. In a previous study, a highly invasive pancreatic cancer cell line was established and found to feature enhanced interleukin-32 (IL-32) expression. However, whether IL-32 promotes the invasiveness by enhancing or suppressing the expression of IL-32 through regulating downstream molecules was unclear. To investigate the effect of IL-32, cells were established with high levels of expression or downregulated IL-32; their invasive ability was measured using a real-time measurement system and the expression of some candidate downstream molecules involved in invasion was evaluated in the two cell types. The morphological changes in both cell types and the localization of IL-32 expression in pancreatic cancer tissues were studied using immunohistochemistry. Among the several splice variants of IL-32, cells transfected with the $\varepsilon$ isoform had increased invasiveness, whereas the IL-32-suppressed cells had reduced invasiveness. Several downstream molecules, whose expression was changed in the two cell types, were monitored. Notably, changes of E-cadherin, CLDN1, CD44, CTGF and Wnt were documented. The morphologies of the two cell types differed from the original cell line. Immunohistochemically, the expression of IL-32 was observed only in tumor cells and not in normal pancreatic cells. In conclusion, IL-32 was found to promote the invasiveness of pancreatic cancer cells by regulating downstream molecules.
\end{abstract}

\section{Introduction}

Pancreatic cancer is a major neoplasm with a high mortality and its prevalence is increasing worldwide. Various efforts have been made to improve its diagnosis and treatment, but the prognosis is poor and the 5-year survival rate is only appproximately $4 \%(1,2)$.

Correspondence to: Dr Johji Imura, Department of Diagnostic Pathology, Faculty of Medicine, Academic Assembly, University of Toyama, 2630 Sugitani, Toyama 930-0194, Japan

E-mail: imura@med.u-toyama.ac.jp

Key words: pancreatic cancer cell, invasion, interleukin-32, epithelial mesenchymal transition, E-cadherin
The reason for the poor prognosis is that the pancreas is a deeply located organ, symptoms are not immediately apparent, and early detection therefore is difficult. Moreover, surgery is impossible due to the invasion into the surrounding organs at the time of onset. On the other hand, the biological properties of pancreatic cancer are characteristic and the tumors have more abundant fibrous stroma than other neoplasms (3). Despite the high degree of this so-called 'desmoplastic change', pancreatic cancer cells are characterized by readily invading the fibrous stroma (4).

Various cell signaling pathways are involved in the invasion mechanism of pancreatic cancer. For example, it has been suggested that several cytokines belonging to the transforming growth factor- $\beta$ family, such as bone morphogenetic protein, contribute to its regulation; these molecules are involved in the epithelial-mesenchymal transition (EMT) and cancer-stroma interaction $(5,6)$. Recent findings suggested that interleukin-32 (IL-32) is deeply involved in the invasiveness of pancreatic cancer, based on a comprehensive gene analysis of highly invasive pancreatic cancer lines subcloned by the invasion assay (IA) method (7). In this study, the gene and protein expression levels of IL-32 were distinctly increased in highly invasive cell lines.

To obtain invasiveness, cancer cells must first be motile and able to disrupt the surrounding hard interstitium, resulting in EMT. EMT is one of the processes that occurs in the embryonic period, as a result of various signaling pathways, but is also a phenomenon observed in tumor tissues (8). EMT cells lose the expression of molecules that cause epithelial binding, produce mesenchymal substances such as vimentin and $\alpha$-smooth muscle actin, and acquire invasiveness (9). It is crucial to elucidate the factors involved in this invasion mechanism that occur early in pancreatic cancer cells. Although there are a few reports available on the relationship between IL-32 expression and pancreatic cancer $(10,11)$, there is, at present, no report that IL-32 has a direct relationship with invasiveness. Therefore, the aim of the present study was to determine, by regulating the expression of the $I L-32$ gene, whether IL-32 expression contributes to the invasiveness of pancreatic cancer and through what molecular mechanism the invasive ability is obtained. Due to the large number of molecules potentially involved in the invasion mechanism, representatives from those involved in EMT and subsequent processes were selected, including E-cadherin, Claudin-1, Slug, CD44 and CTGF as EMT-related molecules (12-14), 
MMP2 and MMP9 as a molecule disrupting the surrounding interstitium (15), and Wnt5a/b and transglutaminase (TGM) 2 for cell motility (16-18).

\section{Materials and methods}

Cell lines and cell culture. The human pancreatic cancer cell lines (PANC-1, MIA PaCa-2) were obtained from the American Type Culture Collection. Cells with high invasive potential were established from PANC-1 cells by the IA method, as described in a previous report (7). The original cell line was used as the parent $(\mathrm{P})$ and the subcloned cell line that had acquired high invasiveness was referred to as (S). Each cell line was maintained in RPMI-1640 culture medium (FUJIFILM Wako Pure Chemical Corporation) containing $10 \%(\mathrm{v} / \mathrm{v})$ fetal bovine serum, $100 \mathrm{IU} / \mathrm{ml}$ penicillin and $100 \mu \mathrm{g} / \mathrm{ml}$ streptomycin at the condition of $37^{\circ} \mathrm{C}$ and $5 \% \mathrm{CO}_{2}$.

cDNA cloning and overexpression of human IL-32. The method used to establish cells in which IL-32 is continuously highly expressed is mentioned below. Using the first-strand cDNA derived from MIA PaCa-2 cells with low invasive potential as a template, human IL-32 cDNA was amplified by PCR with primers 5'-ggggtaccGCCATGTGCTTCCCGAAGGTC CTCTCTG-3' (the KpnI site is underlined) and 5'-gggcggccgc TCATTTTGAGGATTGGGGTTCAGAG-3' (the NotI site is underlined) and subcloned into pLITMUS 28 plasmid vector (New England Biolabs). After confirmation of the cDNA sequence using an ABI3500 Genetic Analyzer (Thermo Fischer Scientific, Inc.), the $K p n \mathrm{I}-$ and NotI-digested IL-32 $\beta$ and IL-32 $\varepsilon$ cDNA fragments were cloned between the KpnI and NotI sites of the pEBMulti-Neo vector (FUJIFILM Wako Pure Chemical Corporation), to yield the plasmids pEB-IL-32 $\beta$ and pEB-IL-32 $\varepsilon$. Then, the pEB-IL32 $\beta$, pEB-IL32 $\varepsilon$ and control pEB Multi-Neo plasmids were transfected into $P$ cells with Lipofectamine 3000 reagent (Thermo Fischer Scientific, Inc.), according to the manufacturer's protocol. After $48 \mathrm{~h}$, the transfected P cells were selected with G418 $(500 \mu \mathrm{g} / \mathrm{ml})$ for 2 weeks, yielding IL-32 $\beta$-overexpressing ( $\beta$ ), IL-32ع-overexpressing $(\varepsilon)$ and control cells with the unmodified plasmid (MOCK).

Small interfering (si) RNA-mediated downregulation of IL-32 expression. For the RNA interference assay, a targeted small interfering RNA (siRNA) oligonucleotide was obtained from the Nippon gene. The sequences of the IL-32 siRNA were as follows: Sense, 5'-GGGAGAGCUUUUGUGACAAdTdT-3'; anti-sense, 5'-UUGUCACAAAAGCUCUCCCdTdT-3'. As a negative control, the si sequences for Luciferase were as follows: sense, 5'-CGUACGCGGAAUACUUCGATT-3'; anti-sense, 5'-UCGAAGUAUUCCGCGUACGTT-3'. In particular, $\mathrm{S}$ and $\mathrm{P}$ cells were transfected with $20 \mathrm{nM}$ of each siRNA using Lipofectamine ${ }^{\text {TM }}$ RNAiMAX Transfection Reagent (Thermo Fischer Scientific, Inc.) to knock down the $I L-32$ gene, and then incubated for a further $24 \mathrm{~h}$ at $37^{\circ} \mathrm{C}$. S cells transfected with IL-32 siRNA were referred to as S-si cells and the negative control cells were denoted as S-siLuci, and they were used for the invasion assay. P cells transfected with IL-32 siRNA were referred to as P-si cells and the negative control cells were denoted as P-siLuci cells and were used for the measurement of mRNA and protein expression levels.
Construction of genome editing plasmids to induce mutations in IL-32 loci by CRISPR/Cas9. pX362 was used for genome editing of the IL-32 locus (19). After digestion of pX362 with BbsI, the oligonucleotides 5'-caccTGATGACATGAAGAAGCTGA-3' and 5'-aaacTCAGCTTCTTCATGTCATCA-3' (capitals and small letters are protospacer sequences and additional sequences to clone into the $B b s I$ site, respectively) corresponding to the single guide RNA target sequence in the exon 3 of $I L-32$, were annealed and subcloned into the BbsI site of pX362 to yield pX362IL-32-1.

Establishment of IL-32 knockout cell lines. To establish knockout cells in which the expression of IL-32 was continuously arrested the pX362IL32-1 plasmid, in which the IL-32 sequence was mutated, was transfected into P cells using Lipofectamine 3000 reagent (Thermo Fischer Scientific, Inc.). The DNA-transfected P cells were selected with puromycin (Thermo Fischer Scientific, Inc.; $2 \mu \mathrm{g} / \mathrm{ml}$ ) for $48 \mathrm{~h}$ and incubated for 2 weeks. Then, these were cloned individually by limiting dilution and clones in which IL-32 mRNA expression was appropriately suppressed were selected. Finally, a knockout cell line of IL-32 (ko) was established.

RNA isolation. Cytoplasmic RNA was extracted using a PureLink ${ }^{\circledR}$ RNA Mini Kit (Thermo Fischer Scientific, Inc.) from sub-confluent cells. Concentrations of RNA were measured with a NanoDrop 1000 (Thermo Fisher Scientific, Inc.).

Selection of the downstream molecules related to $I L-32$ and validation of expression changes by western blot analysis $(W B)$ and reverse transcriptase-quantitative polymerase chain reaction ( $R T-q P C R)$

$W B$. Protein extraction was performed using a Complete Lysis-M (Roche) from sub-confluent cells. Concentrations were calculated using the Bradford method. After adjusting $18 \mu \mathrm{g}$ of each protein sample was separated by sodium dodecyl sulfate-polyacrylamide gel electrophoresis with $4-15 \%$ gradient gels, and transfected to PVDF membrane Trans-Blot Turbo Transfer Pack (Bio-Rad) using Trans-Blot Turbo Blotting System (Bio-Rad). The membranes were blocked with 5\% skim milk at room temperature for $1 \mathrm{~h}$, and then treated with each primary antibody at $4^{\circ} \mathrm{C}$ overnight. Primary antibodies were as follows: anti-IL-32 (1:500; cat. no. 11079-1-AP; ProteinTech Group, Inc.), anti-E-cadherin (cat. no. 3195), anti-CD44 (cat. no. 3570), anti-GAPDH (cat. no. 5174), anti- $\beta$-actin (cat. no. 3700), anti-Wnt5a/b (cat. no. 2530) (all 1:1,000; all from Cell Signaling Technology) and anti-Claudin (1:100; cat. no. ab15098; Abcam) and anti-MMP2 (cat. no. ab92536), anti-MMP9 (cat. no. ab137867), anti-Slug (cat. no. ab27568), anti-CTGF (cat. no. ab6992) (all 1:1000 dilution; and all from Abcam) and anti-TGM2 (diluted 1:2000; cat. no. ab2386; Abcam). After washing, the membranes were reacted with the secondary antibody at room temperature for $1 \mathrm{~h}$. Secondary antibodies were horseradish peroxidase-conjugated sheep anti-rabbit IgG (cat. no. 7074S) or anti-mouse IgG (cat. no. 7076S; 1:10000; Cell Signaling Technology). Detection and visualization were performed with an ImageQuant LAS500 (GE Healthcare) system using ECL Select Western Blotting Detection Reagent (GE Healthcare) as the chemiluminescence detection reagent.

$R T-q P C R$. RNA was extracted as aforementioned. cDNA was obtained from the extracted RNA using a Transcriptor 
Universal cDNA Master Kit (Roche). Next, quantitative PCR was conducted using a FastStart Essential DNA Green Master (Roche). Primer sequences for IL-32 amplification were as follows: sense 5'-AGCTGGAGGACGACTTCAAA-3'; anti-sense 5'-AGAGCA GCAGAAACTCTGGA-3'. Primer sequences for internal reference ( $\beta$-actin) were as follows: sense 5'-CTGGAACGGTGAAGGTGACA-3'; anti-sense 5'-AAGGGACTTCC TGTAACAATGCA-3'. Each cDNA and specific primer were adjusted to final concentrations of $2.5 \mathrm{ng} / \mu \mathrm{l}$ and $500 \mathrm{nM}$, respectively, and then mixed in a total volume of $20 \mu \mathrm{l}$ of FastStart Essential DNA Green Master reagent. The qPCR reaction was carried out in a LightCycler $^{\circledR} 480$ (Roche) with setting denaturation at $95^{\circ} \mathrm{C}$ for $10 \mathrm{sec}$, annealing at $60^{\circ} \mathrm{C}$ for $10 \mathrm{sec}$, and extension at $72^{\circ} \mathrm{C}$ for $15 \mathrm{sec}$ per cycle, with 45 cycles. The analysis was conducted using LightCycler Nano Software (Roche). The relative expression levels of mRNA were calculated based on quantitative cycles (Cq) and using the relative $\mathrm{Cq}\left(2^{-\Delta \Delta \mathrm{Cq}}\right)$ method (20).

Real-time monitoring of invasion ability. To evaluate the invasive ability of the cells (MOCK, $\beta, \varepsilon, \mathrm{S}$-siLuci, S-si, P, $\mathrm{ko})$, invasion was measured in real-time using the $\mathrm{xCelligence}$ system (ACEA Biosciences), as reported previously (7).

Morphological and immunohistochemical studies. Studies of the morphological changes of $\mathrm{P}, \varepsilon, \beta$, and ko cells were carried out by observing living cells in culture with an inverted microscope (Olympus IX70; Olympus Corporation). An immunohistochemical study analyzed $10 \%$ buffered formalin-fixed for $<2$ days at room temperature and paraffin-embedded sections of pancreatic ductal adenocarcinoma tumors, surgically resected and obtained from University Hospital of Toyama. The samples comprised normal pancreatic tissue and invasive lesions. Immunohistochemistry was performed using a BenchMark GX automated IHC/ISH slide staining system (Roche). Deparaffinized sections $(4 \mu \mathrm{m})$ sections were heat-treated for antigen retrieval at $98^{\circ} \mathrm{C}$ for $30 \mathrm{~min}$ and then incubated with $1: 100$ IL-32 antibody at room temperature for $1 \mathrm{~h}$. Incubated sections were visualized by diaminobenzidine reaction for $2 \mathrm{~min}$.

Ethics approval was obtained from the University Hospital of Toyama Ethics Committee (no. R2020054). Although consent was not obtained from each patient, the patients were notified of the details of the study by opt-out consent and had the right to refuse participation in the study.

\section{Results}

$m R N A$ and protein expression levels of IL-32 in the cells overexpressing $I L-32$. Cells constitutively expressing IL-32 were prepared by transfection of the splice variants of IL-32, IL-32ع and IL-32 $\beta$, into P cells. The expression level of IL-32 mRNA was markedly increased in both $\beta$ and $\varepsilon$ cells, compared with MOCK cells (Fig. 1A). At the protein level, it was confirmed that both $\varepsilon$ and $\beta$ cells had enhanced expression, reflecting the difference in the molecular weights of the translated proteins (Fig. 1B).

The mRNA and protein expression levels of IL-32 with siRNA transfection and knockout of IL-32. The mRNA expression in $P$ cells was knocked down using IL-32 siRNA transfection or knockouts following the CRISPR-cas9 method. The expression
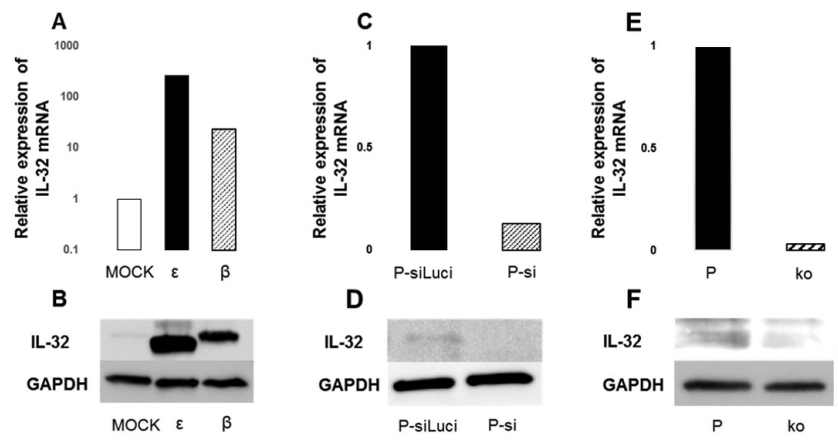

Figure 1. Expression of IL-32 mRNA and protein. (A and B) Upregulation of IL-32, (C and D) downregulation of IL-32 by knockdown with siRNA, and (E and F) by knockout.
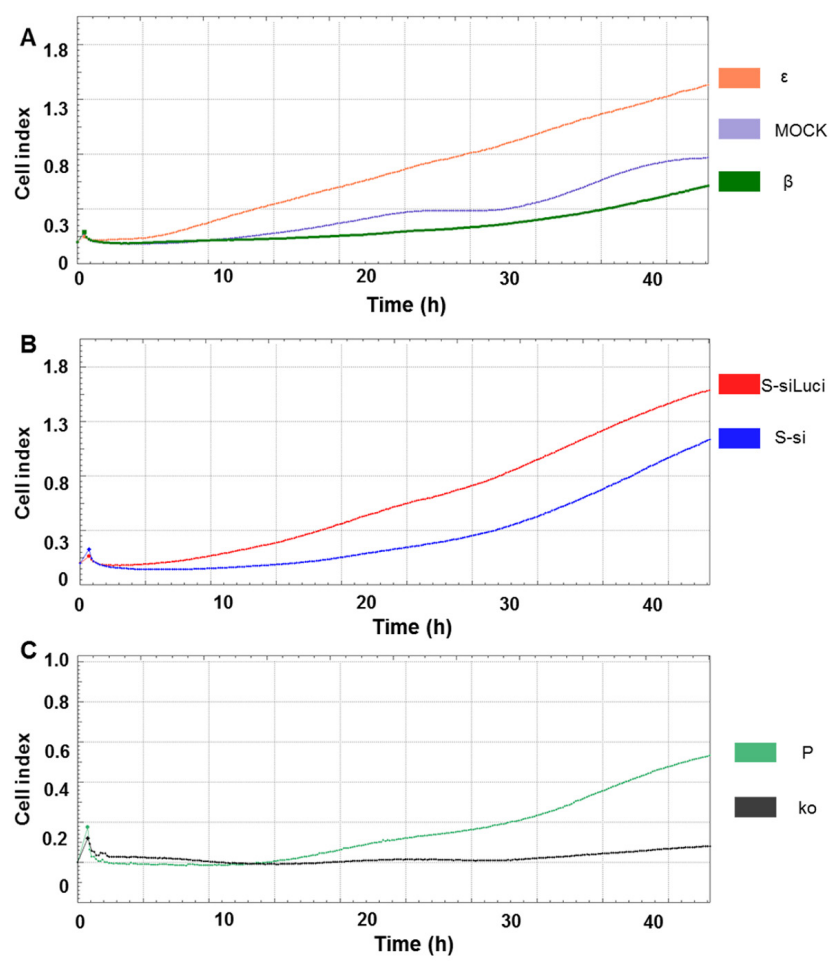

Figure 2. Time-course changes of the invasiveness in IL-32. (A) Upregulated, (B) knockdown, and (C) knockout cells.

of IL-32 mRNA was reduced in both P-si and ko cells (Fig. 1C and E). In P-si cells, the expression level of IL-32 mRNA was reduced to $1 / 5$ of that of P-siLuci (Fig. 1C). However, the effect of the siRNA decreased after approximately 4 days and the expression levels gradually recovered (data not shown). By contrast, the expression of IL-32 mRNA was suppressed to $\sim 1 / 32$ in ko cells, compared with P cells (Fig. 1E). Furthermore, the IL-32 mRNA expression was persistently suppressed and did not recover, even after more than one week (data not shown). Protein expression of IL-32 was almost completely suppressed in ko cells (Fig. 1F).

Changes in invasiveness with overexpression and suppression of $I L-32$. The number of invading $\varepsilon$ cells gradually increased immediately after the beginning of the analysis and they continued to be invasive even after $45 \mathrm{~h}$. $\beta$ cells invaded slowly, compared to $\varepsilon$ cells and their tendency for invasion was similar to MOCK cells (Fig. 2A). S-si cells began to invade slowly 

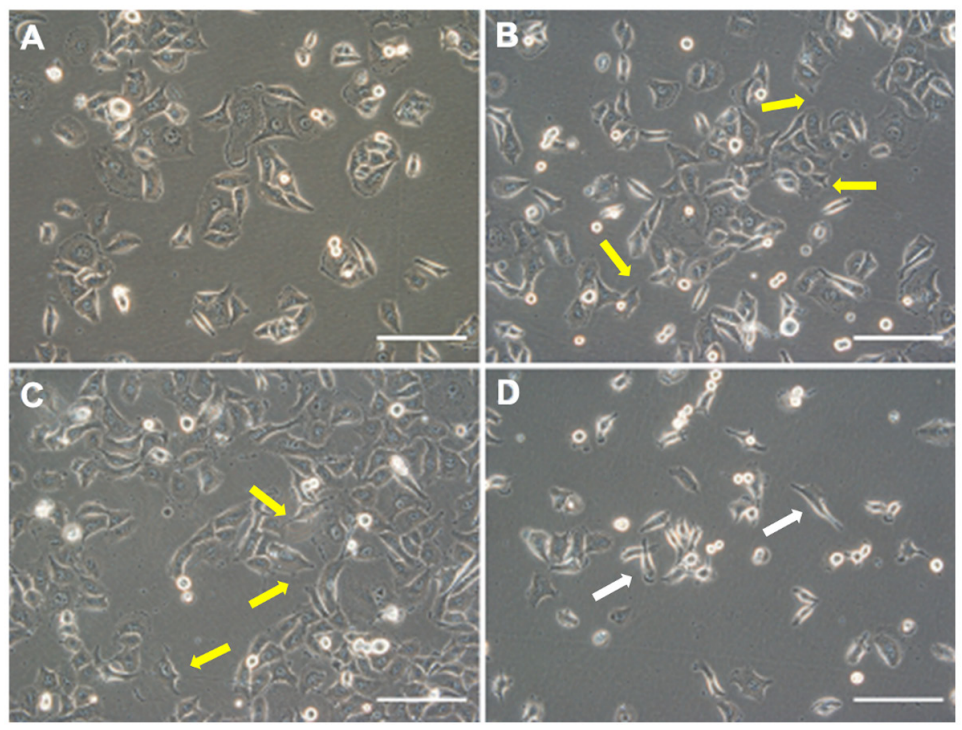

Figure 3. Inverted microscopic findings. (A) Original parental cells, (B) cells with upregulated IL-32ع and (C) IL-32 $\beta$, and (D) IL-32 knockout cells. Yellow arrows indicate characteristic structure of protrusion and white arrows indicate spindle-shaped cells with poor protrusions. Scale bar indicates $100 \mu \mathrm{m}$.

A

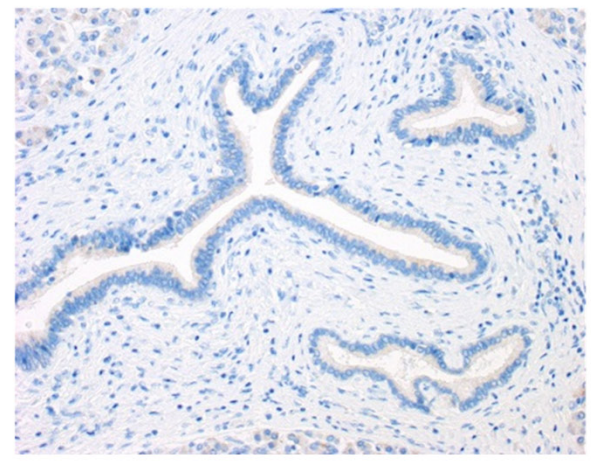

B

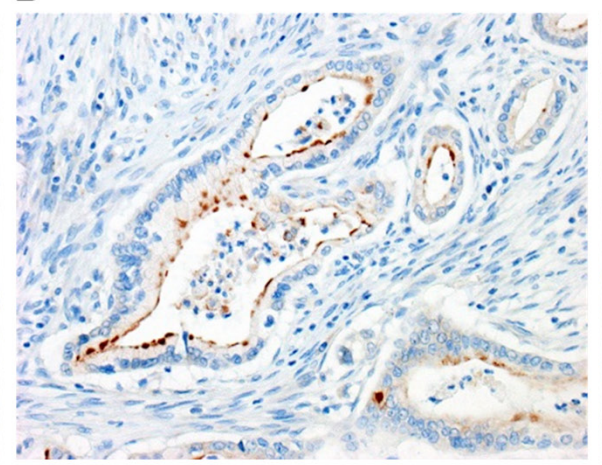

C

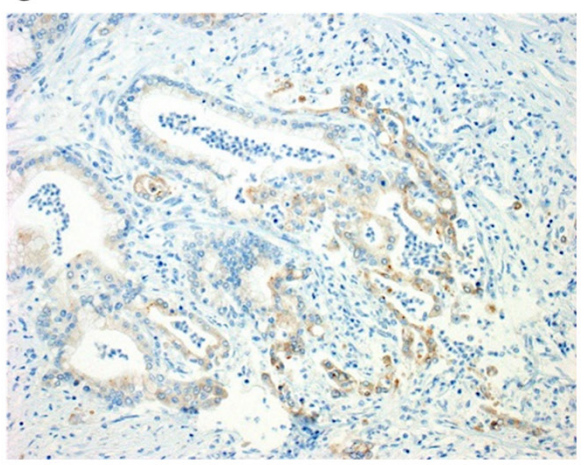

Figure 4. Immunohistochemical findings in resected tissues. (A) Normal pancreatic duct, (B) cancer cells, and (C) invading cells in the invasive front.

after $20 \mathrm{~h}$ and the number of invading cells was lower than that of the S-siLuci control (Fig. 2B). Suppression of IL-32 expression in S-si is shown in Fig. S1. The ko cells had considerably reduced invasiveness and almost no invasive cells were observed, even after $50 \mathrm{~h}$ (Fig. 2C).

Morphological and immunohistochemical study of the IL-32 overexpressing and suppressed cells. Morphological changes to the overexpressing and suppressed cells are shown in Fig. 3. $\beta$ and $\varepsilon$ cells had a more abundant cytoplasm than P cells and cells with a polygonal shape were predominant. Furthermore, prominent lamellipodia or filopodia were observed in many cells (Fig. 3A-C). By contrast, ko cells had a narrowed cytoplasm and the morphology was spindle-shaped. Furthermore, the formation of lamellipodia was poor, these being composed of cells with one or two filopodia (Fig. 3D).

To confirm the expression of IL-32 in pancreatic cancer tissues, an immunohistochemical study was performed using surgically resected materials (Fig. 4A-C). No staining of IL-32 was observed in normal pancreatic tissue (Fig. 4A). By contrast, immunostaining of IL-32 was evident in the cytoplasm of the differentiated adenocarcinoma cells, mainly along the luminal side (Fig. 4B). As a characteristic finding, many tumor cells, predominantly in the invasive front, showed a positive reaction (Fig. 4C).

Molecules affected by IL-32. To determine the signaling pathway through which IL-32 exerts its function, the expression of several proteins was examined by WB analysis. Distinct differential expression of some molecules was observed (Fig. 5). The most apparent change was in E-cadherin, an adherent junction factor. Its expression was markedly decreased in $\varepsilon$ and $\beta$ cells, while an increased expression was found in ko cells. Thus, the expression patterns of IL-32 and E-cadherin were opposed. By contrast, Claudin-1, a tight junction protein, tended to be upregulated in $\varepsilon$ and $\beta$ cells and suppressed in ko cells, showing a pattern correlated with the expression of IL-32. MMP2 expression tended to be increased in $\varepsilon, \beta$ and ko cells, while MMP9 was decreased in $\varepsilon$ and ko cells. The low molecular component of Slug was increased only in $\varepsilon$ cells. Although the expression of CD44 was similarly enhanced in ko cells, no distinct difference in expression was observed in $\varepsilon$ and $\beta$ cells. There was no distinct difference in the expression of Wnt $5 \mathrm{a} / \mathrm{b}$ in the ko and $\beta$ cells but a marked 


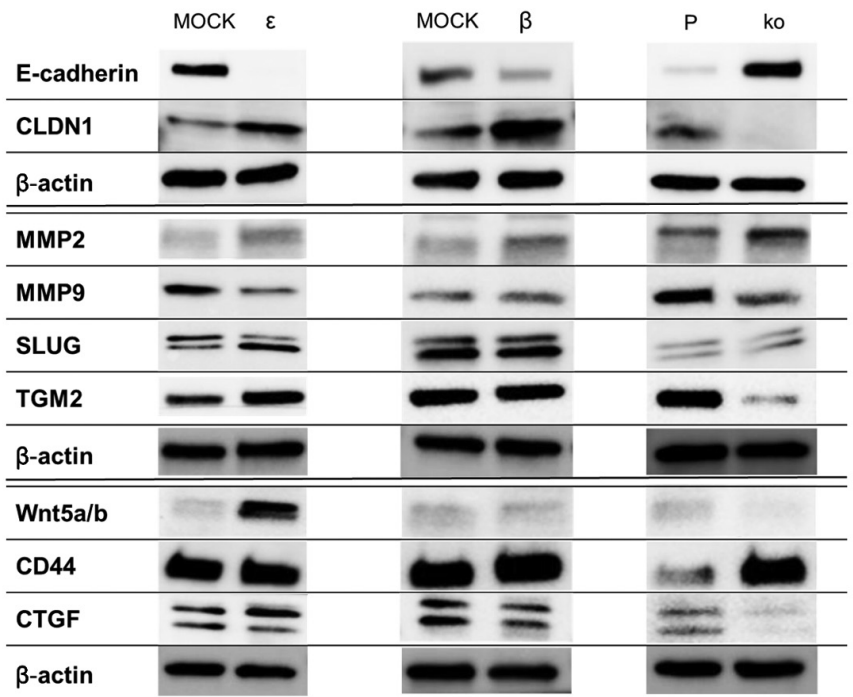

Figure 5. Western blot analysis of the invasion-associated proteins, IL-32-upregulated cells $(\varepsilon, \beta)$ and IL-32 knockout cells (ko). Expression pattern were affected by altered expression of IL-32, especially, the expression pattern of E-cadherin was inversely associated with the expression of IL-32.

upregulation was observed in $\varepsilon$ cells. The expression of CTGF and TGM2 was suppressed in ko cells, which did not differ distinctly from $\varepsilon$ and $\beta$ cells.

\section{Discussion}

IL-32 is a cytokine that has been attracting attention in recent years for its association with the development of various tumors (21-26). Previously, we established a highly invasive human pancreatic cancer cell line and investigated comprehensively the factors that enhance its invasiveness (7). Findings of that study showed that IL-32 is particularly involved in invasiveness (7). Therefore, the aim of the present study was to determine in more detail the relationship between IL-32 and invasiveness in pancreatic cancer. In particular, it was of interest whether the invasiveness was altered by enhancing or suppressing the expression of IL-32. IL-32 is known to have several splice variants (23). In this study, cells overexpressing IL-32 were established using the IL- $32 \varepsilon$ and IL-32 $\beta$ isoforms and it was confirmed that their expression was distinctly enhanced in the respective transfected cells, compared with P cells. However, siRNA-transfected, P-si, and ko cells, were generated with suppression of IL-32. For the ko cells, a mutant plasmid constructed for the exon 3 region, which is present in all of these isoforms of IL-32, was designed and transfected into the cells. Comparing ko and P-si cells to P cells, the expression level of IL-32 mRNA was reduced to $<20 \%$ in P-si cells but was reduced to $5 \%$ or less in ko cells. The suppression of IL-32 expression was maintained for one week or longer in the ko cells but for only about 4 days in the siRNA-transfected cells. The ko cells were judged to be capable of prolonged maintenance of IL-32 suppression.

A comparison of the invasiveness of these IL-32 high-expressing and suppressed cells is shown in Fig. 2A-C. IL-32 high-expressing $\varepsilon$ cells, which were transfected with the IL-32 $\varepsilon$ isoform, showed a tendency for increased invasiveness compared with MOCK. The $\varepsilon$ cells invaded from an early-stage and this property increased rapidly (Fig. 2A). Conversely, $\beta$ cells showed less invasiveness, similar to MOCK. This difference in invasive ability may be due to the lack of exon 4 in the structure of the IL-32 $\varepsilon$ isoform (23), or it may be the difference in the ability of either isoform to regulate downstream factors. That is, exon 4 may have a function of suppressing invasion ability. However, currently, there are few reports on $\varepsilon$ and invasion; thus, an experimental system in which exon 4 is selectively deleted must be prepared and examined. By contrast, S-si and ko cells have reduced or an absence of invasion, compared with the original cells. From these experimental results, the different invasiveness of these highly expressing and suppressed cells also support the concept that IL-32 is a key molecule involved in invasiveness.

Differences were also observed among $\varepsilon, \beta$, and ko cells in terms of cell morphology. Both $\varepsilon$ and $\beta$ cells had abundant cytoplasm, compared with P cells, and the cells had a polygonal shape and actively formed lamellipodia and filopodia. These characteristic morphological changes are observed not only in tumor cells but also in normal cells when they migrate. When the cells move, the protrusions elongate by the polymerization of intracellular actin (27). It is presumed that lamellipodia play a key role in the advance of invasive cancer cells in vivo (27); thus, in that respect, it may be considered that this motility is the reason $\varepsilon$ cells have become highly invasive. The findings that lamellipodia and filopodia were observed in $\varepsilon$ cells with high invasiveness may also support these speculations. The morphological changes in ko cells, which showed almost no invasion, also may provide complementary evidence for the involvement of IL-32 in motility. The immunohistochemical analysis also indicated that IL-32 was involved in the invasiveness of pancreatic cancer cells, in that it was expressed only in pancreatic cancer cells and it was not detected in normal pancreatic tissue. Furthermore, the expression of IL-32 was observed in many tumor cells at the invasive fronts, where invasiveness is considered to be most active, supporting the concept that IL-32 is expressed mainly in actively invading cells.

Next, the involvement of downstream factors under the control of IL-32 was investigated. This comparative study of $\varepsilon, \beta$, and ko cells focused on several molecules that have been suggested to be involved in invasion and changes were detected in the expression of some of the molecules. The expression of E-cadherin and IL-32 showed an inverse correlation. Expression of E-cadherin mRNA and protein was decreased in $\varepsilon$ and $\beta$ cells but was increased in ko cells. Thus, IL-32 may be an upstream factor that regulates the expression of E-cadherin (21). Tumor cell budding from the cell nest is generally seen in the early stages of the invasion of epithelial tumors, due to the loss of adhesion between the cells. E-cadherin is an adherent junction factor and plays an important role in cell-to-cell adhesion. When this molecule is lost or attenuated, the adherence between the tumor cells is reduced and tumor cells are released from the cell nest (27). From these findings, it seems that expression of IL-32 is important at the early stage of invasion and reduces the expression of E-cadherin. In addition, there are cell biological features of pancreatic cancer that are rarely seen in other malignant tumors. Although tumor cells may also infiltrate the desmoplastic stroma as solitary cells, another characteristic finding is that the tumor cells 
may invade in a glandular arrangement, with cell polarity (4). Claudin-1, a tight junction protein, may be responsible for these changes; it is essential for the cell polarity that maintains the glandular structure, as well as the barrier mechanism (28). This study also showed a positive correlation of the expression of IL-32 and Claudin-1.

Molecules such as MMPs, that destroy the basement membrane and stroma, play a major role in invasion. Among the many MMPs, it has been reported that MMP2 and MMP9 are important in the invasion process and are highly expressed by many invading tumor cells. Although there are many molecules that regulate MMPs, the present study has shown a partial correlation between these MMPs and IL-32 $(24,26,29)$. It is possible that the splice variant isoforms of IL-32 regulate their expression via another pathway. A similar tendency was observed for Slug, which is usually expressed during transformation into mesenchymal cells in the EMT phenomenon (27). Herein, only the $\varepsilon$ isoform of IL-32 increased Slug expression. Therefore, only this isoform regulates Slug expression in the mechanism of invasion and/or EMT in pancreatic cancer.

IL-32 is believed to act upstream of the STAT3 (30), NF- $\mathrm{B}$ (29), and Akt pathways (26) in guiding EMT and angiogenesis. In the present study, the focus was on the Wnt signaling pathway, which may be heavily involved in invasiveness, cell motility and cell polarity. At present, there are no reports of the relationship between IL-32 and Wnt in neoplastic disease. If IL-32 acts upstream of Wnt, the Wnt pathway may be involved in the regulation of invasiveness, cell motility and cell polarity by IL-32. However, Wnt5a/b was induced only in $\varepsilon$ cells. This finding suggests that the highly invasive $\varepsilon$ isoform of IL-32 may regulate the Wnt pathway. In the future, it will be important to study changes of $\beta$-catenin and RhoA, which form part of this pathway. It was reported previously that $\beta$-catenin was also highly expressed in gastric cancer cells overexpressing IL-32 (24). In particular, considering that $\beta$-catenin is also markedly involved with E-cadherin, this may be the key to explaining the present finding of a negative correlation between IL-32 and E-cadherin.

Regarding other molecules involved in tumor cell invasion, CD44 also has various splice variants and is involved in cell-to-cell adhesion or cell-to-cell matrix adhesion (31). Although a relationship with IL-32 has not been reported, speculating from the finding that CD44 expression is enhanced in ko cells, IL-32 may suppress CD44 expression, resulting in decreased adhesion. CTGF also has essential roles in many biological processes, including cell adhesion, migration, proliferation and angiogenesis, and is critically involved in several neoplasms (32). TGM is also involved in tumor cell migration, invasion, and proliferation (33). Both molecules were also slightly increased when $\varepsilon$ of IL-32 was highly expressed, whereas the expression of the two molecules was decreased in ko cells. This finding suggests that IL-32 may play a role as an upstream factor regulating these molecules.

In conclusion, IL-32 was found to be an important molecule that regulates the invasiveness of pancreatic cancer. Previously, we reported the establishment of a highly invasive cell line from pancreatic cancer and a comprehensive expression gene analysis revealed that IL-32 is highly expressed in these cells. Herein, more detail is provided of IL-32 function and its role in the invasion process. The results showed that the invasiveness of pancreatic cancer cells could be controlled by regulating the expression of IL-32. Furthermore, it was revealed that IL-32 is an important cytokine, as an upstream factor that regulates various molecules involved in cell adhesion, motility, and invasion. If low-molecular-weight chemical compounds or antibodies that specifically bind to IL-32 could be created in the future, it may be possible to suppress tumor cell invasion by their local administration into the lesion. As a result, metastasis may be suppressed, and this may be an approach leading to an improvement in patient prognosis.

\section{Acknowledgements}

pSpCas9n(BB)-2A-Puro (PX462) was a gift from Professor Feng Zhang (Broad Institute of Massachusetts Institute of Technology and Harvard, Cambridge, MA, USA) (Addgene plasmid no. 48141). The authors would like to thank Ms. Noriko Matsugi (Department of Molecular Neuroscience, Faculty of Medicine, Academic Assembly, University of Toyama, Japan) for their technical help.

\section{Funding}

This research was supported by the Japan Society For The Promotion Of Science (JSPS) Grants-in-Aid for Scientific Research (KAKENHI), grant no. 16K08707.

\section{Availability of data and materials}

The analyzed data sets derived from this study are available from the corresponding author on reasonable request.

\section{Authors' contributions}

KT, AS and JI designed the study, performed the experiment and analyzed data. AS and JI confirm the authenticity of all the raw data. HM provided advice and technical support concerning construction of IL-32 overexpression strain and knockout strain. AN, ST, TM and TNa advised on the construction of the study design and added critical review of the manuscript. TNi and $\mathrm{HH}$ performed collection, analysis and interpretation of some experimental data. KT and JI wrote the manuscript.

\section{Ethics approval and consent to participate.}

This research was approved by the University Hospital of Toyama Ethics Committee (no. R2020054). Consent was not obtained from each patient, albeit the patients were notified of the details of the study by opt-out consent and had the right to refuse participation in the study. All research procedures were carried out in accordance with the Declaration of Helsinki.

\section{Patient consent for publication}

Not applicable.

\section{Competing interests}

The authors declare that they have no competing interests. 


\section{References}

1. Vincent A, Herman J, Schulick R, Hruban RH and Goggins M: Pancreatic cancer. Lancet 378: 607-620, 2011.

2. Kleeff J,Korc M, Apte M,La Vecchia C, Johnson CD, Biankin AV, Neale RE, Tempero M, Tuveson DA, Hruban RH, et al: Pancreatic cancer. Nat Rev Dis Primers 2: 16022, 2016.

3. Pelosi E, Castelli G and Testa U: Pancreatic Cancer: Molecular Characterization, Clonal Evolution and Cancer Stem Cells. Biomedicines 5: 65, 2017

4. Hruban RH, Pitman MB and Kimstra DS: Tumors of the Pancreas, Afip Atlas of Tumor Pathology. 6th edition. American Registry of Pathology, Washington, DC, pp 1201-126, 2007.

5. Luo J, Chen XQ and Li P: The Role of TGF- $\beta$ and Its Receptors in Gastrointestinal Cancers. Transl Oncol 12: 475-484, 2019.

6. Miyazono K, Katsuno Y, Koinuma D, Ehata S and Morikawa M: Intracellular and extracellular TGF- $\beta$ signaling in cancer: Some recent topics. Front Med 12: 387-411, 2018

7. Takagi K, Imura J, Shimomura A, Noguchi A, Minamisaka T, Tanaka S, Nishida T, Hatta H and Nakajima T: Establishment of highly invasive pancreatic cancer cell lines and the expression of IL-32. Oncol Lett 20: 2888-2896, 2020.

8. Nisticò P, Bissell MJ and Radisky DC: Epithelial-mesenchymal transition: General principles and pathological relevance with special emphasis on the role of matrix metalloproteinases. Cold Spring Harb Perspect Biol 4: 4, 2012.

9. Fuxe J, Vincent T and Garcia de Herreros A: Transcriptional crosstalk between TGF- $\beta$ and stem cell pathways in tumor cell invasion: Role of EMT promoting Smad complexes. Cell Cycle 9 : 2363-2374, 2010

10. Chen J, Wang S, Su J, Chu G, You H, Chen Z, Sun H, Chen B and Zhou M: Interleukin-32 $\alpha$ inactivates JAK2/STAT3 signaling and reverses interleukin-6-induced epithelial-mesenchymal transition, invasion, and metastasis in pancreatic cancer cells. OncoTargets Ther 9: 4225-4237, 2016.

11. Nishida A, Andoh A, Inatomi O and Fujiyama Y: Interleukin-32 expression in the pancreas. J Biol Chem 284: 17868-17876, 2009

12. Kyuno D, Takasawa A, Kikuchi S, Takemasa I, Osanai M and Kojima T: Role of tight junctions in the epithelial-to-mesenchymal transition of cancer cells. Biochim Biophys Acta Biomembr 1863: 183503, 2021.

13. Chen C, Zhao S, Karnad A and Freeman JW: The biology and role of CD44 in cancer progression: Therapeutic implications. J Hematol Oncol 11: 64, 2018.

14. Jacobson A and Cunningham JL: Connective tissue growth factor in tumor pathogenesis. Fibrogenesis Tissue Repair 5 (Suppl 1): S8, 2012.

15. Scheau C, Badarau IA, Costache R, Caruntu C, Mihai GL, Didilescu AC, Constantin C and Neagu M: The Role of Matrix Metalloproteinases in the Epithelial-Mesenchymal Transition of Hepatocellular Carcinoma. Anal Cell Pathol (Amst) 2019: 9423907, 2019

16. Pukrop T, Klemm F, Hagemann T, Gradl D, Schulz M, Siemes S, Trümper L and Binder C: Wnt 5a signaling is critical for macrophage-induced invasion of breast cancer cell lines. Proc Natl Acad Sci USA 103: 5454-5459, 2006.

17. Takeshita A, Iwai S, Morita Y, Niki-Yonekawa A, Hamada M and Yura Y: Wnt $5 \mathrm{~b}$ promotes the cell motility essential for metastasis of oral squamous cell carcinoma through active Cdc42 and RhoA. Int J Oncol 44: 59-68, 2014.

18. Agnihotri N, Kumar S and Mehta K: Tissue transglutaminase as a central mediator in inflammation-induced progression of breast cancer. Breast Cancer Res 15: 202, 2013.
19. Ito T, Hayashida M, Kobayashi S, Muto N, Hayashi A, Yoshimura $\mathrm{T}$ and Mori $\mathrm{H}$ : Serine racemase is involved in d-aspartate biosynthesis. J Biochem 160: 345-353, 2016.

20. Livak KJ and Schmittgen TD: Analysis of relative gene expression data using real-time quantitative PCR and the 2(-Delta Delta C(T)) Method. Methods 25: 402-408, 2001

21. Lee J, Kim KE, Cheon S, Song JH, Houh Y, Kim TS, Gil M, Lee KJ, Kim S, Kim D, et al: Interleukin-32 $\alpha$ induces migration of human melanoma cells through downregulation of E-cadherin. Oncotarget 7: 65825-65836, 2016.

22. Sloot YJE, Rabold K, Ulas T, De Graaf DM, Heinhuis B, Händler K, Schultze JL, Netea MG, Smit JWA, Joosten LAB, et al: Interplay between thyroid cancer cells and macrophages: Effects on IL-32 mediated cell death and thyroid cancer cell migration. Cell Oncol (Dordr) 42: 691-703, 2019.

23. Sloot YJE, Smit JW, Joosten LAB and Netea-Maier RT: Insights into the role of IL-32 in cancer. Semin Immunol 38: 24-32, 2018.

24. Tsai CY, Wang CS, Tsai MM, Chi HC, Cheng WL, Tseng YH, Chen CY, Lin CD, Wu JI, Wang LH, et al: Interleukin-32 increases human gastric cancer cell invasion associated with tumor progression and metastasis. Clin Cancer Res 20: 2276-2288, 2014.

25. Yousif NG, Al-Amran FG, Hadi N, Lee J and Adrienne J: Expression of IL-32 modulates NF- $\mathrm{KB}$ and p38 MAP kinase pathways in human esophageal cancer. Cytokine 61: 223-227, 2013.

26. Zhou Y, Hu Z, Li N and Jiang R: Interleukin-32 stimulates osteosarcoma cell invasion and motility via AKT pathway-mediated MMP-13 expression. Int J Mol Med 35: 1729-1733, 2015.

27. Weinberg RA: The Biology of Cancer. 2nd edition. Garland Science, Cambridge, MA, pp 658-691, 2013.

28. Ikenouchi J, Matsuda M, Furuse M and Tsukita S: Regulation of tight junctions during the epithelium-mesenchyme transition: Direct repression of the gene expression of claudins/occludin by Snail. J Cell Sci 116: 1959-1967, 2003.

29. Zeng Q, Li S, Zhou Y, Ou W, Cai X, Zhang L, Huang W, Huang $L$ and Wang Q: Interleukin-32 contributes to invasion and metastasis of primary lung adenocarcinoma via NF-kappaB induced matrix metalloproteinases 2 and 9 expression. Cytokine 65: 24-32, 2014

30. Zhao WB, Wang QL, Xu YT, Xu SF, Qiu Y and Zhu F: Overexpression of interleukin-32 $\alpha$ promotes invasion by modulating VEGF in hepatocellular carcinoma. Oncol Rep 39: 1155-1162, 2018.

31. Klingbeil P, Marhaba R, Jung T, Kirmse R, Ludwig T and Zöller M: CD44 variant isoforms promote metastasis formation by a tumor cell-matrix cross-talk that supports adhesion and apoptosis resistance. Mol Cancer Res 7: 168-179, 2009.

32. Tsai HC, Su HL, Huang CY, Fong YC, Hsu CJ and Tang CH: Correction: CTGF increases matrix metalloproteinases expression and subsequently promotes tumor metastasis in human osteosarcoma through down-regulating miR-519d. Oncotarget 11: 492, 2020.

33. Wang X, Yu Z, Zhou Q, Wu X, Chen X, Li J, Zhu Z, Liu B and Su L: Tissue transglutaminase-2 promotes gastric cancer progression via the ERK1/2 pathway. Oncotarget 7: 7066-7079, 2016.

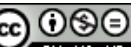

This work is licensed under a Creative Commons Attribution-NonCommercial-NoDerivatives 4.0 International (CC BY-NC-ND 4.0) License. 